\title{
Bovine tuberculosis and brucellosis in cattle and African buffalo in the Limpopo National Park, Mozambique
}

Manfred Tanner ${ }^{1 *}$, Osvaldo Inlameia ${ }^{2}$, Anita Michel $^{3}$, Gabriel Maxlhuza ${ }^{4}$, Alberto Pondja $^{2}$, Jose Fafetine ${ }^{2}$, Balthazar Macucule ${ }^{4}$, Massicame Zacarias ${ }^{4}$, Joaquim Manguele ${ }^{4}$, Ivania Moiane $^{2}$, Angelica Suzana Marranangumbe ${ }^{5}$, Fernando Mulandane ${ }^{6}$, Christine Schönfeld $^{7}$, Irmgard Moser ${ }^{7}$, Paul van Helden ${ }^{8}$, Adelina Machado ${ }^{2}$

\footnotetext{
${ }^{1}$ Institute for Infectious Diseases and Zoonoses, Ludwig-Maximilians-University, Munich, Germany / International Animal Health Team, Friedrich-Loeffler-Institute, Federal Research Institute for Animal Health, Isle of Riems, Germany

${ }^{2}$ Veterinary Faculty, Eduardo Mondlane University, Maputo, Mozambique.

${ }^{3}$ Department of Veterinary Tropical Diseases, Faculty of Veterinary Science, University of Pretoria, South Africa

${ }^{4}$ National Department of Veterinary Services, Ministry of Agriculture, Maputo, Mozambique.

${ }^{5}$ Wildlife Department, Institute for Agriculture Research of Mozambique, Maputo, Mozambique.

${ }^{6}$ Centre for Biotechnology, Eduardo Mondlane University, Maputo, Mozambique.

${ }^{7}$ Institute of Molecular Pathogenesis, Friedrich-Loeffler-Institute, Federal Research Institute for Animal Health, Jena, Germany.

${ }^{8}$ DST/NRF Centre of Excellence for Biomedical Tuberculosis Research/MRC Centre for Molecular and Cellular Biology, Division of Molecular Biology and Human Genetics, Faculty of Health Sciences, Stellenbosch University, Tygerberg, Western Cape, South Africa

*These authors contributed equally to this work

${ }^{\S}$ Corresponding author, m.tanner@1mu.de
}

\section{Summary}

Bovine tuberculosis (BTB) and brucellosis are prevalent in buffaloes of the Kruger National Park (KNP, South Africa). Both diseases were considered to have no or a very low prevalence in wildlife and livestock in and around the Limpopo National Park (LNP, Mozambique). The same applies for tuberculosis in Gonarezhou National Park (GNP, Zimbabwe), but just recently BTB was detected in buffaloes in the GNP and fears arose that the disease might also spread to the LNP as a result of the partial removal of the fences between the three parks to form the Great Limpopo Transfrontier Park. In order to assess the status of both diseases in and around LNP, 62 buffaloes were tested for bovine tuberculosis (BTB) and bovine brucellosis. The percentage of positive BTB reactors in buffalo was $8.06 \%$ using BovidTB Stat-Pak ${ }^{\circledR}$ and $0 \%$ with BOVIGAM® IFN- $\gamma$ test and IDEXX ELISA. The brucellosis seroprevalence in buffalo was found to be $17.72 \%$ and $27.42 \%$ using Rose Bengal Test (RBT) and ELISA, respectively. In addition, 2,445 cattle in and around the LNP were examined for BTB using the single intradermal cervical comparative tuberculin test (SICCT) and an 
apparent prevalence of $0.98 \%$ was found with no significant difference inside $(0.5 \%)$ and outside $(1.3 \%)$ the park. This is the first published report on the presence of positive reactors to BTB in buffalo and cattle in and outside the LNP. Furthermore we report a marked increase concerning the prevalence of bovine brucellosis in cattle and buffalo. Monitoring the wildlifelivestock-human interface of zoonotic high impact diseases such as BTB and brucellosis is of outmost importance for the successful implementation and management of any transfrontier park that aims to improve the livelihoods of the local communities.

Keywords: buffalo, cattle, wildlife-livestock-human interface, tuberculosis, brucellosis, Limpopo

\section{Introduction}

Bovine tuberculosis (BTB) is considered an exotic disease in sub-Saharan Africa. It is thought to have been introduced to Southern Africa by European cattle herds (Hutcheon, 1880) and it appears unlikely that BTB existed in Southern Africa before the end of the nineteenth century (Henning, 1956;de Garin-Wichatitsky et al. 2013). BTB spilled over from cattle to wildlife, with the African buffalo (Syncerus caffer) now being the major maintenance host in Southern Africa (Paine and Martinaglia, 1929; Bengis et al, 1996; Michel et al, 2006). The spill-over from cattle to buffalo occurred in the Kruger National Park (KNP) most likely already in the 1960s (Michel et al, 2009) when the southern border of the KNP was not fenced, allowing buffalo and cattle to mingle and to use the same pastures and water sources. The disease was detected for the first time in 1990 in an African buffalo (Bengis et al, 1996) and intense monitoring revealed that the disease was spreading northwards and reached the borders to Zimbabwe and Mozambique in 2006. Until the recent detection of BTB in two buffaloes in the southern part of the Gonarhezou National Park (GNP), Zimbabwe has been considered free of the disease (De Garine-Wichatitsky et al, 2010). In some of the southern buffalo herds of the KNP the infection prevalence is as high as 90\% (De Vos et al, 2001; Rodwell et al, 2001).

Even though there are no reports of BTB in Mozambican wildlife species, annual reports of the veterinary services are stating varying prevalences of BTB in cattle of up to $30 \%$, mainly in the provinces of Manica, Sofala, Niassa and Inhambane. A study in Govuro district of Inhambane province even revealed a soaring prevalence of $60 \%$ in cattle from small holding keepers, using single intradermal cervical comparative tuberculin test (SICCT) only 
(Macucule, 2009). However no reports of BTB positive animals have been found in relation to the LNP and surrounding areas.

To our knowledge, only one study regarding the presence of BTB in buffalo and cattle (Pereira et al., 2007) was performed in this area prior to our investigation. The first reliable record of bovine brucellosis in cattle in South Africa dates back to 1906 (Henning, 1956). In the same year the presence of brucellosis in cattle was confirmed in Zimbabwe (Madsen and Anderson, 1995). Serological surveys have revealed that up to $23 \%$ of African buffaloes in KNP are positive for brucellosis (Herr and Marshall, 1981). In Mozambique the first isolation of Brucella abortus was reported by Abreu (1967) investigating cases of abortions in cattle throughout the country. Manhiça (2010) found 2 to 33\% of cattle and small ruminants to be positive for specific antibodies using Rose Bengal, complement fixation, serum agglutination and ELISA tests. However, the prevalence of bovine brucellosis in the buffaloes of the LNP was estimated to be very low (Pereira et al, 2007). African buffaloes are considered a wildlife maintenance host for B. abortus (Godfroid et al, 2010).

The Great Limpopo Transfrontier Park (GLTP, 35,000km²) was founded in December 2002 straddling the borders of South Africa, Mozambique and Zimbabwe, combining the KNP, the LNP and with a corridor the GNP, respectively. Almost 5,000 animals have been translocated from KNP to LNP and $50 \mathrm{~km}$ fence have been dropped, encouraging wildlife to cross borders. However, not only animals will cross borders, but also the pathogens they are carrying. Therefore, diseases that have been previously present only in one or two of the three countries of concern might spread over the entire territory of the recently established transfrontier park and might challenge the success of the peace park.

The main focus of this study was to assess the infection status of buffaloes in the LNP and cattle within and outside the LNP with regard to BTB and brucellosis and the spatial distribution in order to evaluate the impact of the removal of the fences between the KNP and the LNP for the establishment of the GLTP.

\section{Materials and methods}

\section{$\underline{\text { Study design and sampling of buffaloes }}$}

From three buffalo herds that show seasonalmovements migrate between KNP and LNP with an estimated total population size of 250 (Swanepoel, pers. comm.) 62 buffalo have been sampled to perform a tuberculosis field side antibody test based on lateral flow technology (BovidTB Stat-Pak ${ }^{\circledR}$ Chembio Diagnostic Systems, Inc, Massachusetts USA), a blood-based 
tuberculosis-specific interferon gamma $(\mathrm{IFN}-\gamma)$ assay $\left(\mathrm{BOVIGAM}^{\circledR}\right.$, Prionics, Zurich, Switzerland) and a IDEXX ELISA for specific antibodies directed against $M$. bovis. For brucellosis the Rose Bengal Test (RBT, serum agglutination test, Ondersterpoort Biologicals, South Africa) and an ELISA for specific antibodies against B. abortus (Ingezin Brucella Multispecies Compac 2.0, SA, Spain) were performed.

The buffaloes were captured by darting from a helicopter using $6 \mathrm{mg}$ of etorphine hydrochloride $\left({\mathrm{M} 99^{\circledR}}^{\circledR}\right.$, Novartis South Africa Pty Ltd, $9.8 \mathrm{mg} / \mathrm{ml}$ ) combined with $30 \mathrm{mg}$ of xylazine hydrochloride (Xylazil-100, Troy Laboratories Pty Ltd, 10\% concentration) per animal in two different capture operations in 2011. Immobilization drugs were loaded into 1.5 ml Dan-inject ${ }^{\circledR}$ darts fitted with plain Dan-inject ${ }^{\circledR}$ needles $(\mathrm{N} 2030,2.0$ x $30 \mathrm{~mm})$ and delivered using Dan-inject ${ }^{\circledR}$ remote delivery system (dart gun). Jugular vein blood was collected in tubes with clot activator to separate serum and into vacutainer tubes with lithium heparin for recovery of plasma to be used for the IFN- $p$ test. Buffaloes were revived from anesthesia after blood collection using $12 \mathrm{mg}$ of diprenorphine hydrochloride (M5050®, 12 $\mathrm{mg} / \mathrm{ml}$, Novartis South Africa Pty Ltd) combined with $5 \mathrm{mg}$ of atipamezole hydrochloride (Orion Pharma, Orion Corporation Espoo, Finland, 5mg/ml) per animal. Serum and plasma (after stimulation) were transported in liquid nitrogen and stored at $-20^{\circ} \mathrm{C}$ until further use.

\section{$\underline{\text { Study design and sampling of cattle }}$}

From a total population of 34,507 cattle in the district of Massingir (Figure 1), 2,445 from 28 villages were tested by single intradermal cervical comparative tuberculin test (SICCT) representing a sample proportion of $7.09 \%$. The cattle belonged to 894 registered cattle keepers from the Massingir district and 1,337 of the sampled animals lived inside the LNP, whereas 1,108 belonged to villages located in the vicinity, but outside of the park (Table 2). The number of animals per owner tested varied from 1 to 21 depending on the herd size. The sample size in each village was calculated based on the cattle population size, with an expected prevalence of $10 \%$. Calculation was done using the program EPICALC, 2000. The animals were driven into crushes and systematically sampled by using fixed intervals determined by the proportion population size over sample size. All tested animals were marked with numbered metallic ear tags. While reading the tested animals after $72 \mathrm{~h}$, jugular vein blood was collected from a sub-set of 133 animals from 18 villages, including twelve SICCT positive, seven inconclusive and 114 negative reactors in order to compare the SICCT results as reference test with additional serological tests BTB BovidTB Stat-Pak ${ }^{\circledR}$ and 


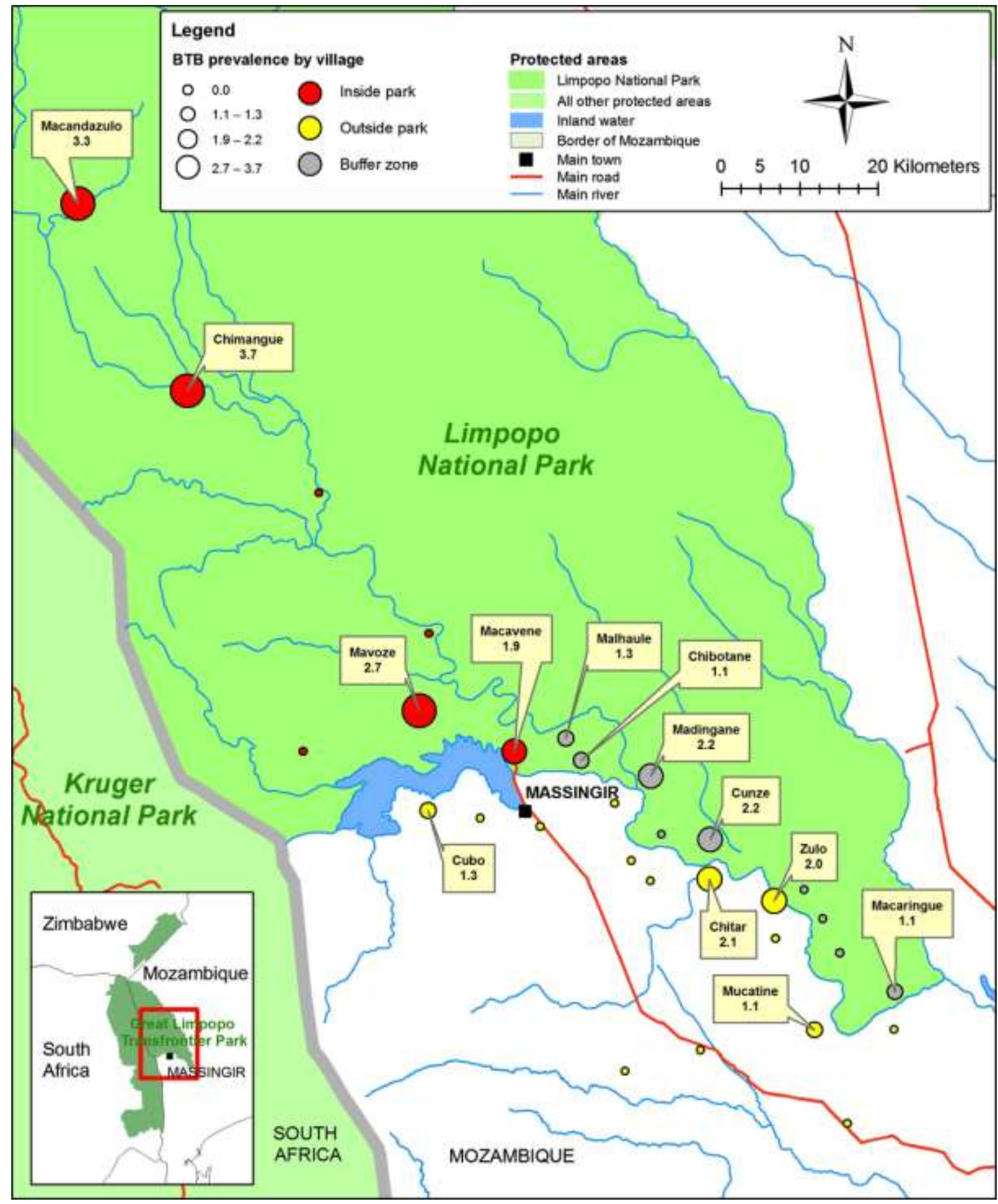

Figure 1. Map of Massingir district showing the BTB prevalences of the villages inside, outside and in the buffer zone of the LNP.

IDEXX ELISA. The serum of the sub-set of 133 animals was also used to test for brucellosis using the RBT. 


\section{Diagnostic systems used to test for BTB}

\section{$\underline{\text { Single intradermal cervical comparative tuberculin test (SICCT) }}$}

The SICCT was applied according to the method recommended by the OIE. Tuberculin was purchased from Symbiotics (Lyon, France) with 2,000 IU for the bovine PPD and 2,500 units for the avian PPD. The date of tuberculin injection, breed, class (calf, steer, heifer, cow, bull, ox) and the owners name were recorded for each animal. The injected animals were then left to mix and graze freely with the rest of the herd. After 72 hours the skin test results were evaluated and recorded. Results were considered positive if the reaction in the skin fold at the site of injection of bovine tuberculin (B72-B0) was $4 \mathrm{~mm}$ or larger than the reaction at the injection site of avian PPD (A72-A0). The reaction was considered inconclusive, when the skin fold measurement was between 2 to $4 \mathrm{~mm}$ and negative if less than $2 \mathrm{~mm}$.

The calculation of BTB prevalence was done using the statistical program SPSS version 14 . The comparison of prevalences according to classes and locations was performed using the $\chi$ square test.

\section{Interferon gamma assay}

For the BOVIGAM ${ }^{\circledR}$ test blood samples were stimulated not later than 10 hours after collection with avian and bovine PPD in order to induce lymphocytes to produce IFN- $\gamma$ and PBS as a negative control. For cell viability control an aliquot of the blood sample was stimulated with pokeweed mitogen. The incubation was performed overnight at $37^{\circ} \mathrm{C}$. After incubation the plasma was harvested and released IFN- $\gamma$ was measured using a sandwich ELISA. Optical density in the reaction wells proportional to the amount of bound IFN- $\gamma$ was quantified using a BIOTEK ELISA reader. Results were obtained by measuring the difference in stimulation (optical densities) between bovine tuberculin PPD and avian PPD following the manufacturer's instructions and positive results were determined as OD450 of PPD-B minus OD450 of PPD-A > 0.1 .

\section{$\underline{\text { BovidTB Stat-Pak® }}$}

To perform the test the serum was brought to room temperature. The test was performed following the instructions of the manufacturer and the test was assessed positive if the blue bands of both, control and test, were readily visible. 


\section{M. bovis antibody test (ELISA)}

The M. bovis antibody test (IDEXX ELISA) was performed following the instructions of the manufacturer IDEXX Laboratories, Inc. Westbrook, Maine, USA. Briefly, $100 \mu 1$ of diluted controls (positive and negative) and serum samples were added into plates coated with $M$. bovis recombinant antigens in duplicated wells. After incubation for $1 \mathrm{~h}$ at room temperature $\left(18-26{ }^{\circ} \mathrm{C}\right)$ the plates were washed 5 times with the washing solution provided in the kit. Subsequently, $100 \mu \mathrm{l}$ of the conjugate (antibody anti-bovine:horseradish peroxidase conjugate) were added to each well and incubated for $30 \mathrm{~min}$ at room temperature. After washing as previously described, $100 \mu \mathrm{l}$ of the substrate (Tetramethylbenzidine - TMB) were added to each well and the plates kept for $15 \mathrm{~min}$ at room temperature. The reaction was stopped after 15 minutes by the addition of $100 \mu \mathrm{l}$ stop solution to each well and the plates read at $450 \mathrm{~nm}$. The presence or absence of antibody to $M$. bovis is determined by calculating the sample to positive $(\mathrm{S} / \mathrm{P})$ ratio for each sample $\left(\mathrm{S} / \mathrm{P}=\frac{\text { sample } A(450)-N C \bar{X}}{P C \bar{X}-N C \bar{X}-}\right)$. Samples with $\mathrm{S} / \mathrm{P}$ ratios greater than or equal to 0.30 are considered positive for $M$. bovis antibodies.

\section{$\underline{\text { Diagnostic systems used to test for bovine brucellosis }}$}

\section{$\underline{\text { Rose Bengal Test }}$}

The test was performed following the instructions of the manufacturer. Briefly, Brucella abortus antigen (VLA, UK) was used to screen sera for the presence of antibodies to Brucella spp. Blood was collected from the jugular vein and after clotting the serum was separated. 25 $\mu l$ of the serum was mixed with the same amount of stained Rose Bengal antigen under $\mathrm{pH}$ 3.65 conditions. Distinct agglutination was considered a positive test.

\section{Enzyme immunoassay (ELISA)}

The blocking enzyme immunoassay (Ingezim Brucella Compac 2.0) was performed following the instructions of the manufacturer (Inmunologia y Genetica Aplicada, SA- Spain). Briefly, $100 \mu \mathrm{l}$ of controls and serum samples were added into plates coated with purified LPS of $B$. abortus in duplicate wells. After incubation for 1 hour at room temperature the plates were washed 4 times with the washing solution provided in the kit. Subsequently, $100 \mu$ of the conjugate (monoclonal antibody against LPS antigen conjugated with peroxidase) were added to each well and incubated for $1 \mathrm{~h}$ at room temperature. After washing as previously described, $100 \mu \mathrm{l}$ of TMB were added to each well and the plates kept for $10 \mathrm{~min}$ at room temperature. The reaction was stopped by the addition of $100 \mu$ stop solution to each well and the plates 
read at $450 \mathrm{~nm}$. Percentage inhibition (PI) calculated for each sample (PI = $100 \mathrm{x}$ [1- (OD sample/OD negative control)] greater than $40 \%$ was considered positive for Brucella antibodies.

\section{Results}

\section{$\underline{\text { Bovine tuberculosis in buffalo }}$}

The results of the tests performed in buffalo samples are presented in Table 1. Five out of 62 buffaloes tested positive in the BovidTB Stat-Pak® test, whereas all animals tested negative with the BOVIGAM gamma interferon and IDEXX ELISA antibody tests.

Table 1. Buffalo results of bovine tuberculosis and brucellosis in LNP

\section{BTB Brucella}

\begin{tabular}{llllll}
\hline \hline Park section & Herd size & Stat-Pak & IFN- $\gamma$ & RBT & ELISA \\
Macandazulu & 103 & $1 / 25$ & $0 / 25$ & $0 / 25$ & $3 / 25$ \\
Chiondziuene & 68 & $1 / 25$ & $0 / 25$ & $6 / 25$ & $9 / 25$ \\
Pafuri & Not known & $2 / 8$ & $\#$ & $4 / 8$ & $4 / 8$ \\
Not known & & & & $1 / 4$ \\
Positive/total number tested & Not known & $1 / 4$ & $\#$ & & $17 / 62(27.42 \%)$ \\
\hline
\end{tabular}

${ }^{a}$ Animals found roaming outside the park, \# test not performed.

\section{Bovine tuberculosis in cattle}

The apparent prevalence of BTB in the Massingir district was estimated as $0.98 \%$ at a $95 \%$ confidence interval (0.64-1.48) with 24 positive and 23 inconclusive skin test results (Table 2). Higher apparent prevalences were found in the villages of Chimangue with $3.7 \%(3 / 81)$, Macandazulo with $3.3 \%$ (3/91) and Mavodze with $2.7 \%$ (3/111). In 15 villages no skin test positive cattle were identified (Figure 1). The comparison of the proportion of positive cattle in the different villages showed no statistical significant differences between them $(p>0.05)$ and the same result was obtained when the proportion of positive cattle resident inside the LNP was compared with the proportion of positive cattle living outside the park (Table 3 ). 
Table 2. Bovine tuberculosis and brucellosis test positive results in cattle in Massingir district

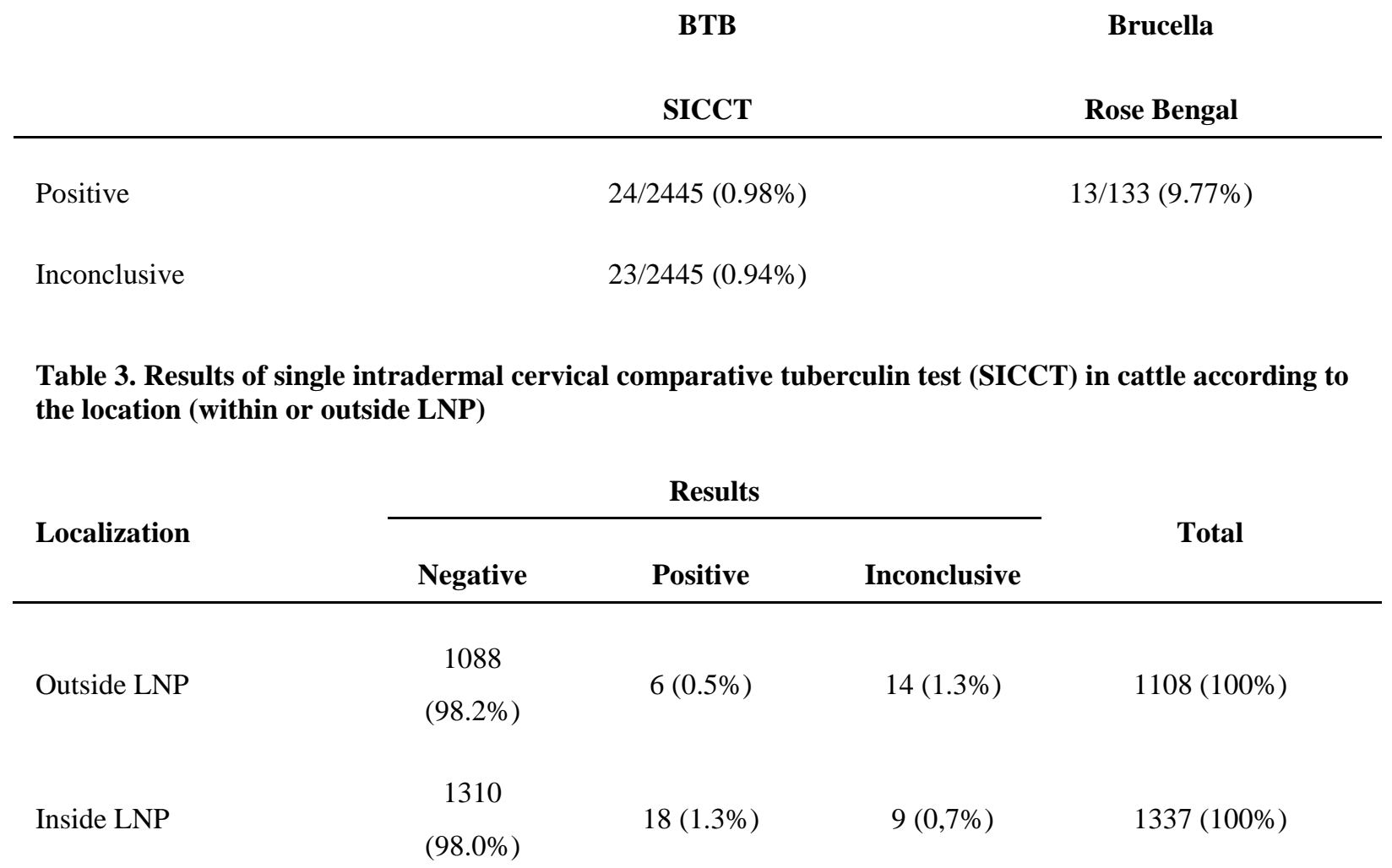

Table 4. Proportion of single intradermal cervical comparative tuberculin test (SICCT) results in cattle according to age and sex

\begin{tabular}{|c|c|c|c|c|}
\hline \multirow{2}{*}{ Class } & \multicolumn{3}{|c|}{ Result } & \multirow{2}{*}{ Total (\%) } \\
\hline & Negative (\%) & Positive (\%) & Inconclusive (\%) & \\
\hline Calf (male) & $17(100)$ & $0(0)$ & $0(0)$ & $17(100)$ \\
\hline Calf (female) & $34(100)$ & $0(0)$ & $0(0)$ & $34(100)$ \\
\hline Steer & 314 (98.7) & $0(0)$ & $4(1.3)$ & $318(100)$ \\
\hline Heifer & $310(99.4)$ & $0(0)$ & $2(0.6)$ & $312(100)$ \\
\hline Bull & 343 (97.2) & $4(1.1)$ & $6(1.7)$ & $353(100)$ \\
\hline Cow & $1148(98.0)$ & $14(1.2)$ & $10(0.9)$ & $1172(100)$ \\
\hline Ox & 219 (96.9) & $6(2.7)$ & $1(0.4)$ & $226(100)$ \\
\hline
\end{tabular}

From the animals tested the majority were cows and no positive cases were found in young animals (Table 4). 
From a sub-set of 133 cattle, $14(10.52 \%), 5(3.76 \%)$ and $12(9.02 \%)$ tested positive using BovidTB Stat-Pak®, IDEXX ELISA and SICCT (reference test), respectively (Table 5). However, a comparison of the results in individual animals showed discrepancies. Out of the 30 positive results in all tests, $11(52 \%)$ were positive with the SICCT only, 13 (40\%) with the BovidTB Stat-Pak ${ }^{\circledR}$ only, and 5 by IDEXX ELISA only, whereas only one animal (1.4\%) was positive in both, the SICCT and BovidTB Stat-Pak® tests and none was positive in all three tests (Table 5).

$\underline{\text { Bovine brucellosis in buffalo }}$

Using the RBT 11 out of $62(17.72 \%)$ animals yielded a positive result and 17 out of 62 $(27.42 \%)$ reacted positive in the ELISA. All positive animals in the RBT were concurrently positive in the ELISA test (Table 1).

\section{Bovine brucellosis in cattle}

Thirteen out of $133(9.77 \%)$ serum samples from cattle tested positive for bovine brucellosis using the RBT (Table 2). This group was tested for bovine tuberculosis as well by SICCT, BovidTB Stat-Pak® and IDEXX ELISA indicating a co-infection only in four animals.

\section{Discussion}

During the last two decades very high BTB prevalences of up to $92 \%$ in buffalo herds in the southern KNP were reported (De Vos et al 2001, Michel et al 2009), and it was estimated that there was an increase of $1.6 \%$ per year from 1991/1992 to 1998 (Rodwell et al, 2001). Introduced to the south of KNP, BTB continued to move northwards, spilled over to various other wildlife species, and was recently found in buffaloes of GNP in Zimbabwe. Following this trend of ongoing dissemination and transmission of the disease it was expected to see an increase of BTB in the vicinity of the LNP over time as $50 \mathrm{~km}$ of buffalo proof fences between RSA and Mozambique were dropped eleven years ago allowing buffaloes and other wildlife to move across borders..

From the 52 buffaloes tested in 2006 using BOVIGAM ${ }^{\circledR}$ only one animal gave a positive result, but was negative in histology and culture results were not available at the time of publication (Pereira et al, 2007). In our study five out of $62(8.06 \%)$ tested positive for BTB using Stat-Pak, but both of the parallel used tests, BOVIGAM ${ }^{\circledR}$ and IDEXX ELISA, produced negative test results and point towards false-positive results of the Stat-Pak test due to crossreactions. On the other hand, due to the low sensitivity $(80-85 \%)$ of the BOVIGAM $^{\circledR}$ test in buffalo (Michel et al, 2011), which might lead to false negative results and the moderate to 
high specificity of the Stat-Pak test (90\%) in buffalo (Michel and Simoes, 2009), which reduces the likelihood of false positive test results, the infection of the buffaloes in the LNP cannot be ruled out.

In cattle we found low BTB prevalences within $(1.3 \%)$ and outside $(0.5 \%)$ the LNP, whereas a study done before in the same area found no cattle or buffaloes, which tested positive for BTB by single tuberculin skin test and culture, respectively. (Pereira et al, 2007). For 133 cattle it was possible to compare skin test, BovidTB Stat-Pak® and IDEXX ELISA results, but even though the first two tests revealed similar numbers of positive results, they were not derived from the same animals (Table 5). Therefore, we speculate that the BovidTB StatPak® might have more value concerning the assessment of the infection status of herds rather than for identifying individual animals infected. The low BTB prevalence in cattle might either indicate an endemic infection status or a recent infection. Acquisition of cattle for restocking from areas, where BTB is highly prevalent is another possibility for the introduction of the disease into the area. However, a recent questionnaire survey revealed no evidence of frequent cattle trade with districts of higher prevalences (data not published). On the other hand there is evidence of exchange of animals between farmers in the district as during the survey animals tested in one village were found in other village as a result of exchange or trade. There was no significant difference in the prevalence of BTB between cattle resident inside or outside the LNP (Figure 1, Table 2). The geographic distribution of the villages with higher BTB prevalences (Figure 1) did not show any tendency that would require a more detailed analysis. We found the highest proportion of positive reactors in oxen (Table 4). Similar results were reported from Tanzania (Kazwala et al, 2001) and from Ethiopia (Dinka and Duressa, 2011). The oxen are trained as draft animals, used in agriculture and transport; thus they have longer life span resulting in increased probability of exposure and to develop the disease. The lack of identification of positive reactors in young animals is consistent with the studies in Tanzania and Ethiopia (Kazwala et al, 2001, Cleaveland et al, 2007); Dinka and Duressa, 2011), where it was evident that the duration of the exposure increased with the age of the animals.

Pereira et al. (2007) studied the occurrence of bovine brucellosis in buffalo in LNP and found one positive case out of 49 buffaloes tested. Several wildlife species have been tested positive for brucellosis in the Kruger National Park with a prevalence of up to $23 \%$ in buffaloes (Herr and Marshall, 1981). In Zimbabwe, a prevalence of even $48 \%$ was found in African buffaloes (Madsen and Anderson 1995). The results of this study of $17.72 \%$ and $27.42 \%$ test positives 
using the RBT and a competitive ELISA, respectively, are perfectly in line with these findings. Only an epidemiological study showing the same molecular well characterised brucella strains in all these countries would suggest also the transmission of the disease across borders.

Even though bovine brucellosis in cattle was found frequently in several Mozambican districts in the past, no surveys have been conducted in the Massingir district before our study, and therefore the previous status of disease is unknown. We found $9.77 \%$ positive reactors in cattle using the RBT (Table 2). Even though the percentage found in buffalo is much higher than the percentage in cattle, it is impossible to predict the direction of spread based on the data currently available.

The rate of brucella infections in humans is virtually unknown and public awareness is extremely low.

\section{Conclusions}

This is the first published report on the possible presence of BTB and bovine brucellosis in buffalo and cattle in and outside the LNP. The infection status of the local communities living in and around the LNP is not yet known.

Zoonotic high impact diseases such as BTB and brucellosis may have a detrimental impact on public health, trade and the socio-economy of these countries. Monitoring the wildlifelivestock-human interface is of outmost importance to authorities and decision makers as there are people residing inside the park and there are no fences to control movements of possibly infected wildlife or livestock.

\section{Acknowledments}

We thank the National Directorate for Veterinary Services of Mozambique and the staff at district level for the support during the study. We also thank the staff of the Serology Laboratory, Eduardo Mondlane University, Mozambique who assisted with serological tests for Brucella abortus. This study was sponsored by the German Research Foundation (DFG). We acknowledge the help of Dr. Mario Ziller, FLI, for supporting the project by providing his knowledge concerning statistical calculations using R DEVELOPMENT CORE TEAM. 2008. R: A language and environment for statistical computing. R Foundation for Statistical Computing, 
Vienna, Austria. ISBN 3-900051-07-0, URL http://www.R-project.org. Finally, we would like to thank Prof Straubinger, LMU, Germany, for reviewing the manuscript.

\section{Author contributions}

MT, IM, OI, AM, participated in the conception and design, the conduct of the study and drafting of the manuscript; JF, BM, ZM, GM, ASM participated in the testing and collection of samples, AP, FM, participated in the analysis of data, AnM, $\mathrm{PvH}$ participated in the design of the study and drafting of the manuscript. All authors critically reviewed the manuscript, read and approved the final version.

\section{References}

Abreu, 1967: Brucelose bovina em Mocambique. Anais dos servicos de veterinaria.Vol 15.

Bengis, R.G., N.P. Kriek, D.F. Keet, J.P. Raath, V. de Vos and H.F. Huchzermeyer, 1996: An outbreak of bovine tuberculosis in a free-living African buffalo (Syncerus caffer sparrman) population in the Kruger National Park: a preliminary report. Onderstepoort J. Vet. Res. 63, $15-18$

Cleaveland, S., D.J. Shaw, S.G. Mfinanga, G. Shirima, R.R. Kazwala, E. Eblate and M. Sharp, 2007: Mycobacterium bovis in rural Tanzania: risk factors for infection in human and cattle populations. Tuberculosis (Edinb), 87, 30-43.

De Garine-Wichatitsky, M., A. Caron, C. Gomo, C. Foggin, K. Dutlow, D. Pfukenyi, E. Lane, S. Le Bel, M. Hofmeyr, T. Hlokwe and A. Michel, 2010: Bovine Tuberculosis in Buffaloes, Southern Africa. Emerg. Infect. Dis. 16, 884-885.

De Garine-Wichatitsky, M., A. Caron, R. Kock, R. Tschopp, M. Munyeme, M. Hofmeyr and A. Michel, 2013: A review of bovine tuberculosis at the wildlife-livestock-human interface in sub-Saharan Africa. Epidemiol. Infect. 141, 1342-1356.

De Klerk-Lorist, L., Bengis, R, 2012: What have we learnt from Bovine Tuberculosis research studies in the buffalo population of the Kruger Park? 5th Annual Kruger Network Meeting.

De Vos, V., R.G. Bengis, N.P. Kriek, A. Michel, D.F. Keet, J.P. Raath and H.F. Huchzermeyer, 2001: The epidemiology of tuberculosis in free-ranging African buffalo (Syncerus caffer) in the Kruger National Park, South Africa. Onderstepoort J Vet Res. 68, 119-130.

Dean, A.S., L. Crump, H. Greter, E. Schelling and J. Zinsstag, 2012: Global burden of human brucellosis: a systematic review of disease frequency. PLoS Negl. Trop. Dis. 6 (10), e1865. 
Dinka, H., Duressa, A, 2011: Prevalence of bovine tuberculosis in Arsi Zones of Oromia, Ethiopia. Afr. J. Agric. Res. 6, 3853-3858.

Fyumagwa, R.D., Wambura P.N., Mellau L.S.B. and Hoare, R., 2009: Seroprevalence of Brucella abortus in buffaloes and wildebeest in the Serengeti ecosystem: A threat to humans and domestic ruminants. Tanzania Vet. J. 26, 62-67.

Godfroid, J., K. Nielsen and C. Saegerman, 2010: Diagnosis of brucellosis in livestock and wildlife. Croat. Med. J. 51, 296-305.

Henning, M.W., 1956: Animal diseases in South Africa, 3rd edn. Central News Agency, Pretoria.

Herr, S. and C. Marshall, 1981: Brucellosis in free-living African buffalo (Syncerus caffer): a serological survey. Onderstepoort J. Vet. Res. 48, 133-134.

Hutcheon, 1880: Annual Report of the Colonial Veterinary Surgeon.

Kazwala, R.R., D.M. Kambarage, C.J. Daborn, J. Nyange, S.F. Jiwa and J.M. Sharp, 2001: Risk factors associated with the occurrence of bovine tuberculosis in cattle in the Southern Highlands of Tanzania. Vet. Res. Commun. 25, 609-614.

Lyashchenko, K.P., R. Greenwald, , J. Esfandiari, M.A. Chambers, J. Vicente, C. Gortazar, N. Santos, M. Correia-Neves, B. M. Buddle, R. Jackson, D.J. O'Brien, S. Schmitt, M.V. Palmer, R.J. Delahay and W.R. Waters, 2008: Animal-side serologic assay for rapid detection of Mycobacterium bovis infection in multiple species of free-ranging wildlife. Vet Microbiol. 132(3-4), 283-92.

Macucule, B.A. 2009: Study of prevalence of bovine tuberculosis in Govuro district, Inhambane province. MSc thesis, University of Pretoria, South Africa.

Madsen, M. and E.C. Anderson, 1995: Serologic survey of Zimbabwean wildlife for brucellosis. J. Zoo Wild Med. 26, 240-245.

Manhica, A.P. 2010: The prevalence of brucellosis in cattle, sheep and goats in Maputo province, Mozambique. MSc thesis, University of Pretoria, South Africa.

Michel, A.L., R.G. Bengis, D.F. Keet, M. Hofmeyr, L.M. Klerk, P.C. Cross, A.E. Jolles, D. Cooper, I.J. Whyte, P. Buss and J. Godfroid, 2006: Wildlife tuberculosis in South African conservation areas: implications and challenges. Vet. Microbiol. 112, 91-100.

Michel, A.L., M.L. Coetzee, D.F. Keet, L. Mare, R. Warren, D. Cooper, R.G. Bengis, K. Kremer and P. van Helden, 2009: Molecular epidemiology of Mycobacterium bovis isolates from free-ranging wildlife in South African game reserves. Vet. Microbiol. 133, 335-343.

Michel, A.L. and M. Simoes, 2009: Comparative field evaluation of two rapid immunochromatographic tests for the diagnosis of bovine tuberculosis in African buffaloes (Syncerus caffer). Vet. Immunol. Immunopathol. 127, 186-189. 
Michel, A.L., B. Müller and P. van Helden, 2010: Mycobacterium bovis at the animal-human interface: Aproblem, or not?. Vet. Microbiol. 140, 371-381.

Michel, A.L., D. Cooper, J. Jooste, L.M. de Klerk, A. Jolles. 2011: Approaches towards optimising the gamma interferon assay for diagnosing Mycobacterium bovis infection in African buffalo (Syncerus caffer). Prev Vet Med. 98(2-3), 142-51.

Paine, R. and G. Martinaglia, 1929: Tuberculosis in Wild Buck Living Under Natural Conditions. J. Comp. Pathol. Therap. 42, 1 - 8.

Pereira, C.L., Costa, R., Mangueze, A. N., Buss. P., Bengis, R., Hofmeyr, M., de Klerk, L., Govender, D., Van Schalkwyk., L, 2007: Results of a survey to detect bovine tuberculosis (BTB), brucellosis and the status of FMD in buffaloes (Syncerus caffer), and tuberculosis in cattle in the Limpopo National Park and adjacent areas. AHEAD-GLTFCA Working Group, 7th meeting, Boane, Mozambique, 13-15.

Rodwell, T.C., N.P. Kriek, R.G. Bengis, I.J. Whyte, P.C. Viljoen, V. de Vos and W.M. Boyce, 2001: Prevalence of bovine tuberculosis in African buffalo at Kruger National Park. J. Wildl. Dis. 37, 258-264.

Swanepoel, B., 2009. Wildlife Manager of Limpop National Park. 